\title{
Transcutaneous medial fixation sutures for free flap inset after robot-assisted nipple-sparing mastectomy
}

\author{
Bong-Sung Kim ${ }^{1,2}$, Wen-Ling Kuo ${ }^{3}$, David Chon-Fok Cheong ${ }^{1}$, Nicole Lindenblatt ${ }^{2}$, \\ Jung-Ju Huang ${ }^{1,4}$ \\ ${ }^{1}$ Division of Reconstructive Microsurgery, Department of Plastic and Reconstructive Surgery, Chang Gung Memorial Hospital, College of \\ Medicine, Chang Gung University, Taoyuan, Taiwan; ${ }^{2}$ Department of Plastic Surgery and Hand Surgery, University Hospital Zurich, Zurich, \\ Switzerland; ${ }^{3}$ Division of Breast Surgery, Department of General Surgery, Chang Gung Memorial Hospital, College of Medicine, Chang Gung \\ University, Taoyuan; ${ }^{4}$ Center for Tissue Engineering, Chang Gung Memorial Hospital, Taoyuan, Taiwan
}

The application of minimal invasive mastectomy has allowed surgeons to perform nipplesparing mastectomy via a shorter, inconspicuous incision under clear vision and with more precise hemostasis. However, it poses new challenges in microsurgical breast reconstruction, such as vascular anastomosis and flap insetting, which are considerably more difficult to perform through the shorter incision on the lateral breast border. We propose an innovative technique of transcutaneous medial fixation sutures to help in flap insetting and creating and maintaining the medial breast border. The sutures are placed after mastectomy and before flap transfer. Three 4-0 nylon suture loops are placed transcutaneously and into the pocket at the markings of the preferred lower medial border of the reconstructed breast. After microvascular anastomosis and temporary shaping of the flap on top of the mastectomy skin, the three corresponding points for the sutures are identified. The three nylon loops are then sutured to the dermis of the corresponding medial point of the flap. The flap is placed into the pocket by a simultaneous gentle pull on the three sutures and a combined lateral push. The stitches are then tied and buried after completion of flap inset.

Keywords Robot-assisted surgery / Mastectomy / Free tissue flaps / Reconstructive surgical procedures

\author{
Correspondence: Jung-Ju Huang \\ Division of Reconstructive \\ Microsurgery, Department of Plastic \\ and Reconstructive Surgery, Chang \\ Gung Memorial Hospital, College of \\ Medicine, Chang Gung University, \\ 5, Fuxing St., Guishan Dist., Taoyuan \\ 33305, Taiwan \\ Tel: +886-3-3281200 (ext.3355) \\ Fax: +886-3-3972681 \\ E-mail: jungjuhuang@gmail.com
}

\begin{abstract}
옹
Bong-Sung Kim was sponsored by the German Research Foundation (Deutsche Forschungsgemeinschaft, KI 1973/2-1) and is a scholarship holder of the Ministry of Foreign Affairs (MOFA), Taiwan.

The authors wish to thank Miss Ingrid Kuo and the Center for Big Data Analytics and Statistics (Grant CLRPG3D0048) at Chang Gung Memorial Hospital for creating the illustrations used herein.
\end{abstract}

\section{INTRODUCTION}

In the search for optimization of female breast reconstruction, minimally invasive procedures offer unprecedented advantages [1]. Minimally invasive nipple-sparing mastectomies (NSMs) mainly encompass endoscopic surgery and robot-assisted oper- ations through a short and inconspicuous incision, usually located in the lateral breast border. When compared to endoscopic NSM, robotic surgery provides better three-dimensional vision for surgeons, enabling more precise dissection and hemostasis, as well as easier and less straining handling [2]. Although the visible cutaneous scars are markedly diminished by the 
aforementioned techniques, they also pose new challenges to reconstructive surgeons, as key steps in free flap reconstruction, such as vascular anastomosis and flap insetting, are significantly complicated. In this article, we will discuss the technique of the senior author $(\mathrm{JJH})$ to optimize flap inset after robot-assisted NSM (R-NSM). Our technique can be applied to the inset of the deep inferior epigastric artery perforator (DIEP) flap, our primary workhorse flap, as well as any other flaps, and helps to shape the medial inferior aspect of the breast border.

\section{IDEA}

The main difficulties in performing microsurgical breast reconstruction after robotic-assisted mastectomy are microsurgical anastomosis via a small incision and transferring the flap into the pocket, insetting it, and shaping it. Via a small lateral incision, it is difficult to push and bring the flap into the mastectomy pocket all the way to reach the most medial inferior point. Even after successfully doing so, the gravity effect remains challenging as the flap may drop inferiorly and laterally with time without a suspension suture. Progressive lateral deviation and medial pole sunken may occur. The idea of performing transcutaneous medial fixation sutures was to both assist in flap transfer and provide suspension fixation of the flap in the medial inferior pole. In order to assist in flap transfer, the sutures are designed first to be dynamic. Suture loops are placed transcutaneously and left inside the pocket without any nodes made. The sutures outside the pocket are temporarily tapped on the chest wall skin. Before flap transfer, the dermis of the flap margin is sutured to the suture loops. By pulling the sutures outside the mastectomy pocket, the sutures first play a guiding role to bring the flap to the most medial inferior pole of the reconstructed breast. After con- firming the location, the sutures are tied to provide permanent fixation of the flap and prevent dropping.

\section{Surgical steps}

The surgical steps prior to the insetting and medial fixation sutures are as follows: (1) sentinel lymph node biopsy, which may be extended to axillary lymph node dissection depending on the intraoperative pathological assessment, and preparation for robotic docking (or another form of minimally invasive mastectomy) through a 4-5 cm skin incision on the anterior axillary line is performed by the breast surgeons. At the same time, the flap (usually a DIEP or alternatively a perforator [profunda artery perforator] flap) is raised by the plastic surgeons, and the pedicle is not transected yet; and (2) R-NSM using the da Vinci Xi surgical system (Intuitive Surgical, Denzlingen, Germany), or any form of minimal invasive mastectomy, is completed.

The surgical steps for the fixation sutures are as follows (Fig. 1, Supplemental Video 1): (1) before vascular anastomosis, the breast skin is marked at 7:00, 7:30, and 8:00 o'clock (left breast) or 4:00, 4:30, and 5:00 o'clock (right breast) at the preferred lower medial border of the breast for the medial suspension sutures; (2) three 4/0 nylon suture loops are placed transcutaneously at the markings on the pocket by a reusable suture needle. The reusable suture needle is removed after the loops are placed inside the pocket; (3) the flap pedicle is transected and anastomosed, usually to the thoracodorsal or lateral thoracic vessels. During anastomosis, the flap is positioned outside on the breast and temporarily fixed to the skin while a second team is closing the donor site. Zone IV and excess flap tissue are excised; (4) deepithelialization of the flap is performed; (5) temporary insetting permits the identification of the three corresponding points of the flap that will be sutured to the 7:00, 7:30, and 8:00

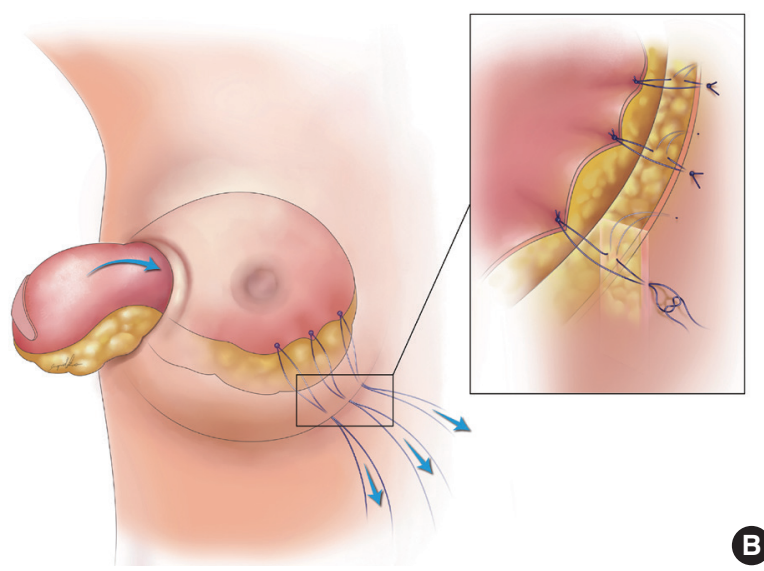

Fig. 1. Schematic draft of the transcutaneous medial fixation sutures. (A) Three nylon sutures are transcutaneously attached at 4:00, 4:30, and 5:00 o'clock (right breast) or 7:00, 7:30, and 8:00 o'clock (left breast) to the deepithelialized flap. The sutures help in the inset process through a small lateral incision in the breast after robot-assisted nipple-sparing mastectomy and in fixing the flap at the medial inferior aspect of the breast. (B) By gently pulling the stitches, the flap is then transferred successfully into the breast pocket. Three knots were made accordingly. 
o'clock or 4:00, 4:30, and 5:00 o'clock positions at the preferred lower medial border of the breast. The points are marked; (6) the three nylon loops are then sutured to the dermis of the marked corresponding medial parts of the deepithelialized flap by two 4/0 nylon sutures per loop; (7) flap is placed into the pocket by a simultaneous gentle pull on the three medial fixation sutures and a lateral push; (8) fixation sutures are tied, cut at the skin level, and buried. Thereby, the mediocaudal breast border is firmly and permanently affixed; or (9) additional measures for breast shaping such as inframammary fold sutures or other fixation sutures may be performed to symmetrize the breast shape to the contralateral site.

\section{DISCUSSION}

A thicker dermis, differences in the cellular and molecular processes of scar maturation, and a significantly higher rate of hypertrophic scars in Asians when compared to Caucasians are well documented [3]. In Asian patient populations, wherein concerns about large and visible scars are widespread, minimally invasive surgery including robotic surgery has therefore gained increasing popularity since its first introduction [4]. R-NSM and immediate microsurgical DIEP flap reconstruction is our primary approach for most patients [5], while the majority of the current literature describes implant-based or pedicled latissimus dorsi flaps for immediate breast reconstruction after RNSM [6-10]. Despite the unquestionable benefits of R-NSM for patients, as recently reported in a large-scale South Korean consortium [11], plastic surgeons face new challenges when it comes to flap inset. Medializing the flap, especially to the inferior medial pole, is extremely difficult via a $4-5 \mathrm{~cm}$ long incision at the lateral breast border. In early microvascular free flap reconstructions after R-NSM, we frequently observed a lateral shift of formerly well-placed flaps, as seen in a 33-year-old patient (Fig. 2) after DIEP flap reconstruction. Correction of this phenomenon via the original incision is difficult once this lateralization is established and even more so when adjuvant radiotherapy has been carried out. Thus, controlled and tailored insetting during reconstruction is of the utmost importance, and recognition of this issue prompted the development of new strategies.

Our approach consists of pre-set transcutaneous permanent sutures (preferred over slowly resorbable sutures due to their durability) that have two purposes. The main purpose is to maintain the mediolateral border of the breast without creating new visible scars. Even if the initially 4-5 cm long incision expands after fixation of the single robotic port and the R-NSM to a certain degree, reaching the medial pocket through it and ap-

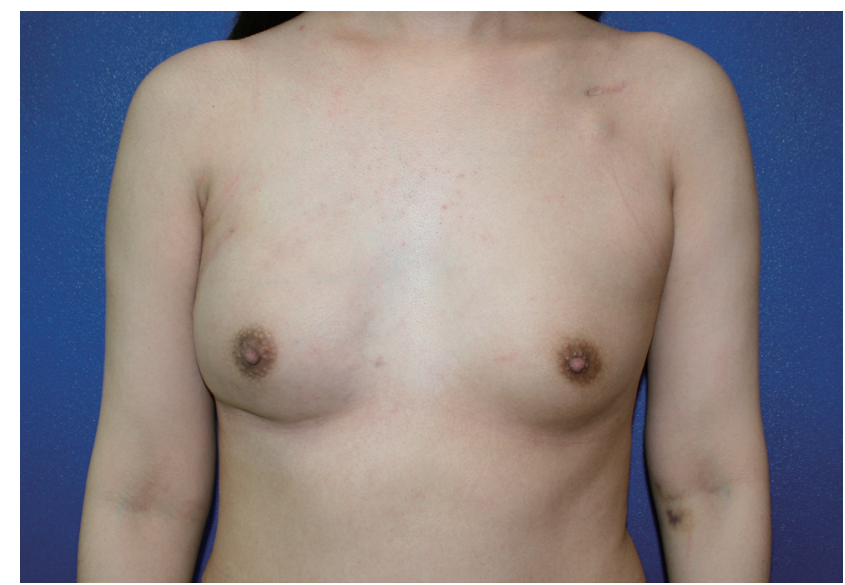

Fig. 2. Lateral shift of the flap without transcutaneous medial fixation sutures (28 weeks after surgery). In this 33-year-old patient, the right breast was reconstructed by a deep inferior epigastric artery perforator flap after robot-assisted nipple-sparing mastectomy due to breast cancer. She also received postmastectomy radiotherapy due to a large tumor size before neoadjuvant chemotherapy. A lateral shift of the right breast with loss of definition of the medial inferior aspect of the breast occurred 28 weeks after surgery.

plying accurate fixation sutures is challenging. Our proposed technique permits an easy, scarless, and precise flap inset.

The second purpose is a simplified insetting of the flap into the pocket without involuntary flap folding by a combination of pull maneuvers on the fixation sutures and pushing the flap through the narrow skin incision into the pocket.

Transcutaneous sutures were earlier applied in plastic surgery, such as for shaping in rhinoplasty [12] or the breast [13], and are used to address inaccessible areas. In this sense, we used a known technique and translated it to the difficult context of breast shaping after robot-assisted mastectomy. In addition, for DIEP flaps, pre-shaping of the flap to the desired cone shape before implanting it into the pocket has been suggested [14], although no work to date has addressed the specific requirements for DIEP inset through a small incision on the lateral thorax as found in our cases.

An obvious concern in our technique is the inevitable creation of retracted scars following the tying of the transcutaneous sutures. However, in our experience, the initially visible retractions start to resolve within 4 weeks and completely disappear after 3 to 4 months, as shown in Fig. 3. In the Taiwanese patient population described in the present manuscript with relatively small flap volumes, transcutaneous anchoring sutures were only needed at the mentioned positions. In larger breasts, additional anchoring sutures may be considered at the upper border of the flap. In these locations, however, we do not recommend transcutaneous anchoring sutures, as these lead to cosmetically unpleasing skin retraction that we have experienced first-hand in 

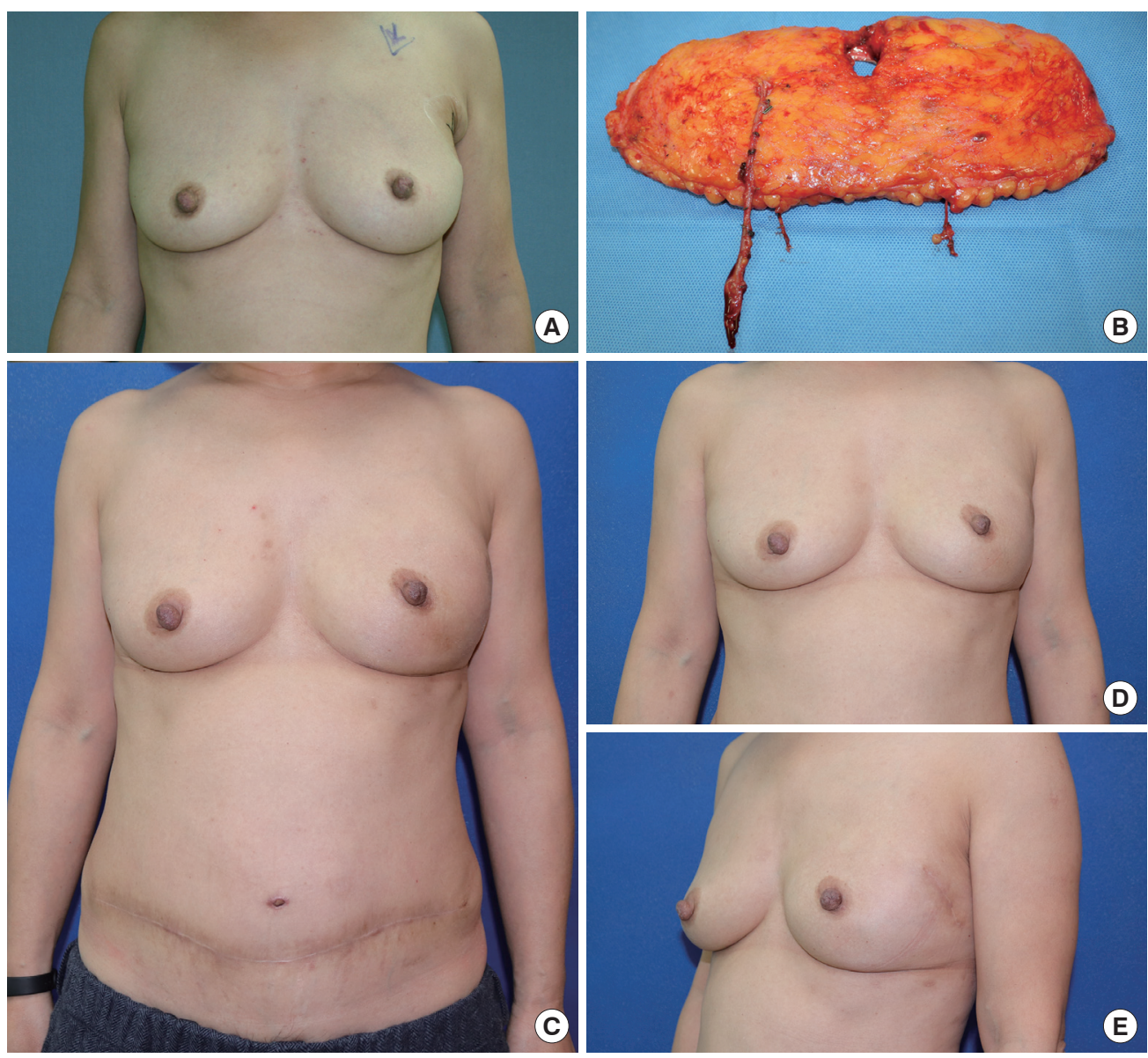

Fig. 3. Patient after a deep inferior epigastric artery perforator (DIEP) flap and transcutaneous medial fixation sutures of the right breast. Breast cancer (ductal carcinoma in situ) of the left breast in this 54-year-old patient was treated with minimally invasive nipple-sparing mastectomy via a small lateral incision and free DIEP flap reconstruction. Her mastectomy specimen weighed $324 \mathrm{~g}$ and a free DIEP was transferred using the thoracodorsal artery and vein as recipient vessels. Before flap inset, transcutaneous medial fixation sutures were placed. The flap, which was de-epithelialized in the buried part, was sutured with the transcutaneous medial fixation suture, and the flap was then pushed into the mastectomy pocket with assistance of pulling from the transcutaneous sutures. (A) Preoperative photo. (B) A free DIEP flap weighing $372 \mathrm{~g}$ was harvested for reconstruction. (C) The initially visible retracted scars over the medial inferior of the left breast began to resolve at a 3-month follow-up examination. The position of the flap was well maintained, without lateral deviation. The donor site scar was also present. (D, E) The anterior and left lateral view of the breast at a postoperative follow-up of 17 months. The scar of the transcutaneous medial fixation suture was completely invisible.

the initial stages. Even with placement of the fixation sutures inside the mastectomy pocket by suturing the flap to the chest wall, retraction may still occur, leading to a disadvantage in terms of cosmesis that could be a permanent problem.

Collectively, we herein propose a simple technique for shaping the medial breast border of free flaps after minimally invasive NSM, including R-NSM. A more elaborate scientific evaluation of our technique is planned once a sufficient number of patient cases and adequate follow-up time are reached.

\section{NOTES}

\section{Conflict of interest}

Jung-Ju Huang is an editorial board member of the journal but was not involved in the peer reviewer selection, evaluation, or decision process of this article. No other potential conflicts of interest relevant to this article were reported.

\section{Ethical approval}

The study was performed in accordance with the principles of the Declaration of Helsinki.

\section{Patient consent}

The patients provided written informed consent for the publication and the use of their images.

\section{Author contribution}

Conceptualization: JJ Huang. Data curation: JJ Huang. Funding 
acquisition: BS Kim. Methodology: WL Kuo, DC Cheong, JJ Huang. Project administration: BS Kim. Visualization: BS Kim, N Lindenblatt. Writing-original draft: BS Kim, JJ Huang. Writing-review \& editing: WL Kuo, DC Cheong, N Lindenblatt, JJ Huang.

\section{ORCID}

Bong-Sung Kim https://orcid.org/0000-0002-0493-3196

Wen-Ling Kuo https://orcid.org/0000-0002-1145-8514

David Chon-Fok Cheong

https://orcid.org/0000-0003-1430-7348

Nicole Lindenblatt https://orcid.org/0000-0003-0293-1004

Jung-Ju Huang https://orcid.org/0000-0001-9568-7834

\section{Supplementary material}

Supplemental Video 1. Video of the transcutaneous medial fixation sutures of a deep inferior epigastric artery perforator flap on the right breast in a 42-year-old patient after robot-assisted nipple-sparing mastectomy. The operating surgeon is the senior author $(\mathrm{JJH})$. Supplemental data can be found at: https://doi. org/10.5999/aps.2021.00843.v001

\section{REFERENCES}

1. Donnely E, Griffin MF, Butler PE. Robotic surgery: a novel approach for breast surgery and reconstruction. Plast Reconstr Surg Glob Open 2020;8:e2578.

2. Park HS, Lee J, Lee DW, et al. Robot-assisted nipple-sparing mastectomy with immediate breast reconstruction: an initial experience. Sci Rep 2019;9:15669.

3. Kim S, Choi TH, Liu W, et al. Update on scar management: guidelines for treating Asian patients. Plast Reconstr Surg 2013;132:1580-9.

4. Toesca A, Peradze N, Manconi A, et al. Robotic nipple-sparing mastectomy for the treatment of breast cancer: feasibility and safety study. Breast 2017;31:51-6.

5. Kuo WL, Huang JJ, Huang YT, et al. Robot-assisted mastectomy followed by immediate autologous microsurgical free flap reconstruction: techniques and feasibility in three different breast cancer surgical scenarios. Clin Breast Cancer 2020;20:e1-8.

6. Toesca A, Peradze N, Galimberti V, et al. Robotic nipplesparing mastectomy and immediate breast reconstruction with implant: first report of surgical technique. Ann Surg 2017;266:e28-30.

7. Struk S, Leymarie N, Honart JF, et al. Robotic nipple-sparing mastectomy with immediate reconstruction by robotically harvested latissimus dorsi muscle in a single position: cadaveric study. J Plast Reconstr Aesthet Surg 2018;71:7646.

8. Sarfati B, Struk S, Leymarie N, et al. Robotic nipple-sparing mastectomy with immediate prosthetic breast reconstruction: surgical technique. Plast Reconstr Surg 2018;142:6247.

9. Lai HW, Chen ST, Lin SL, et al. Robotic nipple-sparing mastectomy and immediate breast reconstruction with gel implant: technique, preliminary results and patient-reported cosmetic outcome. Ann Surg Oncol 2019;26:42-52.

10. Houvenaeghel G, Cohen M, Ribeiro SR, et al. Robotic nipple-sparing mastectomy and immediate breast reconstruction with robotic latissimus dorsi flap harvest: technique and results. Surg Innov 2020;27:481-91.

11. Ryu JM, Kim JY, Choi HJ, et al. Robot-assisted nipple-sparing mastectomy with immediate breast reconstruction: an initial experience of the Korea Robot-Endoscopy Minimal Access Breast Surgery Study Group (KoREa-BSG). Ann Surg 2020 Sep 15 [Epub]. https://doi.org/10.1097/SLA. 0000000000004492.

12. Turhal G, Benzer M, Sahin FF, et al. Transcutaneous suture tip plasty: the technique and outcomes. Ann Otol Rhinol Laryngol 2019;128:789-94.

13. Nakajima Y, Kondoh S, Nishioka $H$, et al. A new percutaneous method for inframammary fold reconstruction in implant-based breast reconstruction: vertical pendulum suture. Medicine (Baltimore) 2018;97:e11964.

14. Odobescu A, Keith JN. Preshaping DIEP flaps: simplifying and optimizing breast reconstruction aesthetics. Plast Reconstr Surg 2021;147:1059-61. 